\title{
A frequency spectrum-based processing framework for the assessment of tree root systems
}

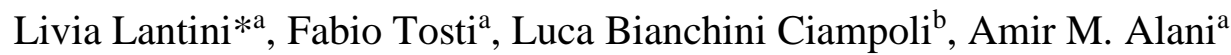 \\ aSchool of Computing and Engineering, University of West London (UWL), St Mary's Road,

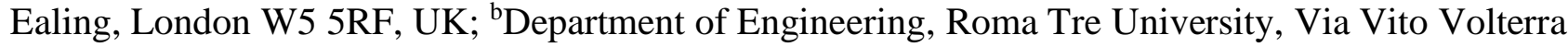 \\ 62, 00146 Roma, Italy
}

\begin{abstract}
The monitoring and conservation of natural assets are nowadays increasingly important, as the emergence of unknown pathogens endangers tree survival. In this respect, root systems are affected by fungal infections that cause root rot and ultimately contribute to the death of trees. Such diseases can quickly spread to surrounding trees and affect wider areas. Since these decays do not usually show visible symptoms, early identification is key to the protection of trees.

Within this framework, non-destructive testing (NDT) methods are becoming increasingly popular, as they are faster and more flexible than destructive methods. Specifically, ground penetrating radar (GPR) is emerging as an accurate geophysical tool for tree roots mapping. Recent research has focused on the implementation of automated algorithms for root mapping in a $3 \mathrm{D}$ environment and the investigation of root mass density.

This research aims to investigate the changes occurring in the frequency spectrum of the GPR signal during root system surveys. To this extent, advanced signal processing techniques (both in time and frequency domain) are applied, to eliminate noise-related information and the disturbance induced by the presence of other features (i.e. pavement layers or underground utilities). The proposed processing framework can be applied for expeditious analyses or on trees difficult to access, where more comprehensive survey methods are not applicable. The results of the application of this methodology to a real-case scenario showed the potential of the applied procedure and will lead to the development of a new approach for investigating root systems using GPR.
\end{abstract}

Keywords: Ground Penetrating Radar (GPR), short-time Fourier transform (STFT), tree root mapping, urban trees

\section{INTRODUCTION}

Trees and forests are invaluable components of humanity and wildlife. Oxygen provision, carbon storage, soil stabilisation, food supply and wildlife habitats are only some of the natural heritage's key benefits ${ }^{1}$. Besides, the impact that trees have on human health and behaviour ${ }^{2,3}$ is a scientifically recorded evidence, as they contribute to noise and pollution mitigation 4,5 and create an enjoyable environment for socialisation ${ }^{6}$.

Of all the tree organs, roots play a vital role in the health of plants and trees. In fact, they anchor the tree to the soil and provide support ${ }^{7}$, absorb soil minerals and water, store nutrients and synthesise hormones ${ }^{1}$. Tree roots adopt stochastic patterns that differ considerably from one tree species to another ${ }^{8,9}$. Besides, tree patterns often relate to the tree's health status and therefore have been extensively used in arboriculture applications as a diagnostic tool ${ }^{10}$.

Different techniques were proposed to effectively map a given tree's root structure, which can be classified into destructive and non-destructive testing (NDT) methods. Excavation, uprooting and profile wall technique fall into the destructive methods category ${ }^{11}$. Such approaches can also inflict permanent damage to the surrounding rhizosphere, apart from being inefficient and not suitable for large-scale forestry applications ${ }^{11-13}$, and hence are not favoured by foresters and tree agents. In comparison, NDT methods can map root patterns efficiently, without interfering with the host material and irreversibly harming the tree. Several NDT methods have been employed for root-mapping, including X-ray tomography, nuclear methods and magnetic resonance ${ }^{14-16}$, acoustic methods and electrical resistivity tomography ${ }^{17}$. Groundpenetrating radar is an NDT method which covers a wide range of applications, such as civil and environmental engineering applications ${ }^{18}$, landmine detection ${ }^{19}$ and archaeology ${ }^{20}$. Due to its ease of use, versatility and high resolution, GPR is an extremely attractive option for forestry applications. As such, GPR is becoming increasingly common amongst foresters and tree officers as an efficient method to non-destructive estimate of root patterns ${ }^{21-23}$. Recent research concentrated on automated root mapping algorithms within $3 \mathrm{D}$ environments ${ }^{24}$. In more depth, these studies focused on evaluating root 
interconnections with nearby trees' root systems ${ }^{25}$, estimating the mass density of tree root systems and improving root detection through advanced GPR signal processing technology ${ }^{26}$. Also, recent studies proved that GPR is an effective tool for mapping the root system's architecture of street trees ${ }^{27}$.

The present study reports the preliminary results of an experimental campaign conducted on a test site located in an urban park in London, United Kingdom. The main aim of this research is to investigate the feasibility of a novel tree root assessment approach, based on the analysis of GPR data both in time and frequency domain. The suggested processing system may be implemented for expeditious analyses or on trees difficult to reach, such as in some urban environments, where more comprehensive survey methods are not applicable. The specific objectives of this research can be outlined as follows: i) understanding the influence of different features (i.e. roots, layers, etc.) on the time-frequency analysis of GPR data; ii) identifying recurring patterns in the data analysis, in order to establish a repeatable data processing methodology.

\section{METHODOLOGY}

\subsection{Test Site and Equipment}

The survey was carried out as part of a major research campaign in Walpole Park, Ealing, London (United Kingdom) (Figure 1). A number of 24 circular scans were performed around the investigated tree, starting $0.50 \mathrm{~m}$ from the bark and spaced $0.30 \mathrm{~m}$ from one another, therefore surveying an overall area of $175.67 \mathrm{~m}^{2}$ around the tree. Among the scans mentioned above, the one located at $2.60 \mathrm{~m}$ from the outer surface of the trunk was selected for analysis purposes.

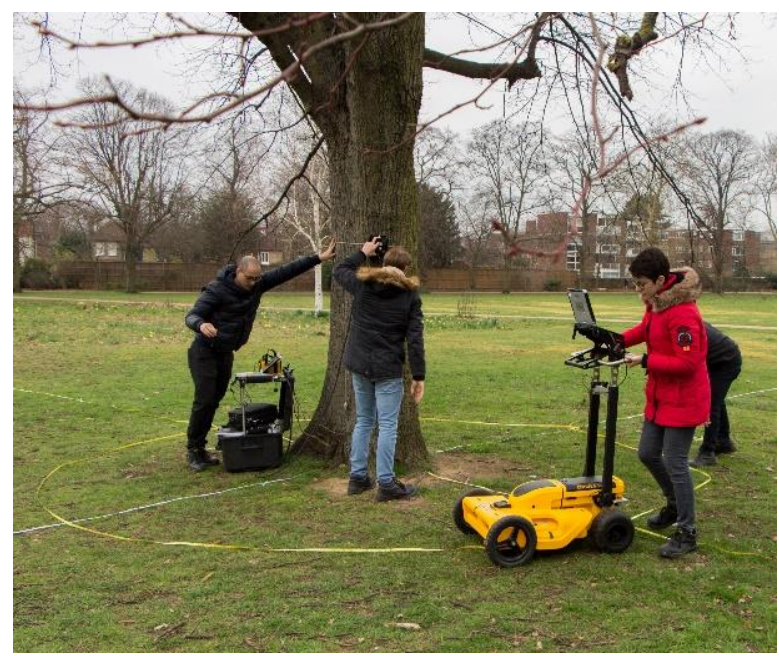

Figure 1. The investigated area

The survey was carried out using the Opera Duo ground-coupled GPR system, manufactured by IDS GeoRadar (Part of Hexagon). The system is equipped with $700 \mathrm{MHz}$ and $250 \mathrm{MHz}$ central frequency antennas. Data were collected using a time window of $80 \mathrm{~ns}$, discretised across 512 samples. The horizontal resolution was set to $3.06 \times 10^{-2} \mathrm{~m}$. In order to achieve the optimal effective resolution, only data collected using the $700 \mathrm{MHz}$ antenna were analysed. This choice was due to the need to achieve a depth of investigation such as to analyse the whole tree root system, without excessively affecting the signal resolution.

\subsection{Data Processing}

This stage aims primarily at reducing information on noise from the GPR data and at obtaining quantifiable information and easily interpretable images for the analysis and interpretation of data. The raw data were hence analysed in both time and frequency domains, following a multi-stage processing procedure ${ }^{28,29}$ :

- Zero-offset removal

- Time-zero correction

- Singular value decomposition (SVD) filter

- $\quad$ Band-pass filtering 
Subsequently, data were processed using a short-time Fourier transform (STFT) ${ }^{30}$. This approach allows data to be analysed in both time and frequency domain, by evaluating how the frequency spectrum changes with time, as follows:

$$
\operatorname{STFT}(t, \omega)=\int_{t}[x(t) \cdot w(\tau-t)] \cdot e^{-j \omega t} d t
$$

where STFT is the frequency energy at time $t$ and frequency $\omega, x$ is the reflected amplitude and $w$ is the window function.

\section{RESULTS AND DISCUSSION}

\subsection{Target Identification and Application Windows}

Figure 2 shows the radargram of the selected scan after the application of the signal processing techniques. Inside, a smaller area was chosen on which to apply the STFT function. Such area was chosen as it includes two relatively isolated hyperbolas of comparable shape, positioned one above the other at a distance of approximately $5 \mathrm{~ns}$ from each other. The two hyperbolas are likely due to the top and bottom reflections of a structural root, with a diameter of about $0.15 \mathrm{~m}$. The root interjects a semi-horizontal layer (presumably a geosynthetic layer), which is visible on both sides of the top hyperbola.

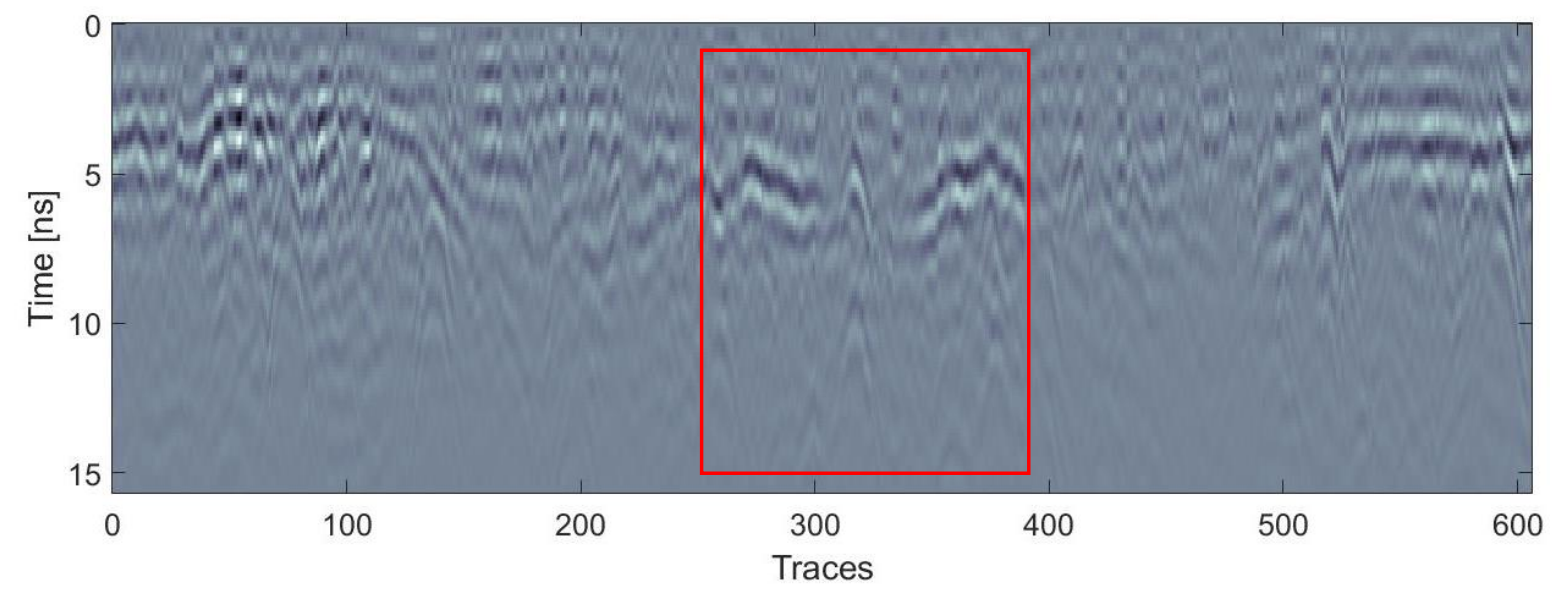

Figure 2. Radargram of the analysed scan. The red square identifies the selected application area.

The selected area was then divided into 5 application windows, as shown in Figure 3. Such windows, tailored to be as wide as the reference hyperbolas, consist of 19 tracks (i.e. A-scan) each, with a width of about $0.55 \mathrm{~m}$ per window, for a total width of $2.75 \mathrm{~m}$.
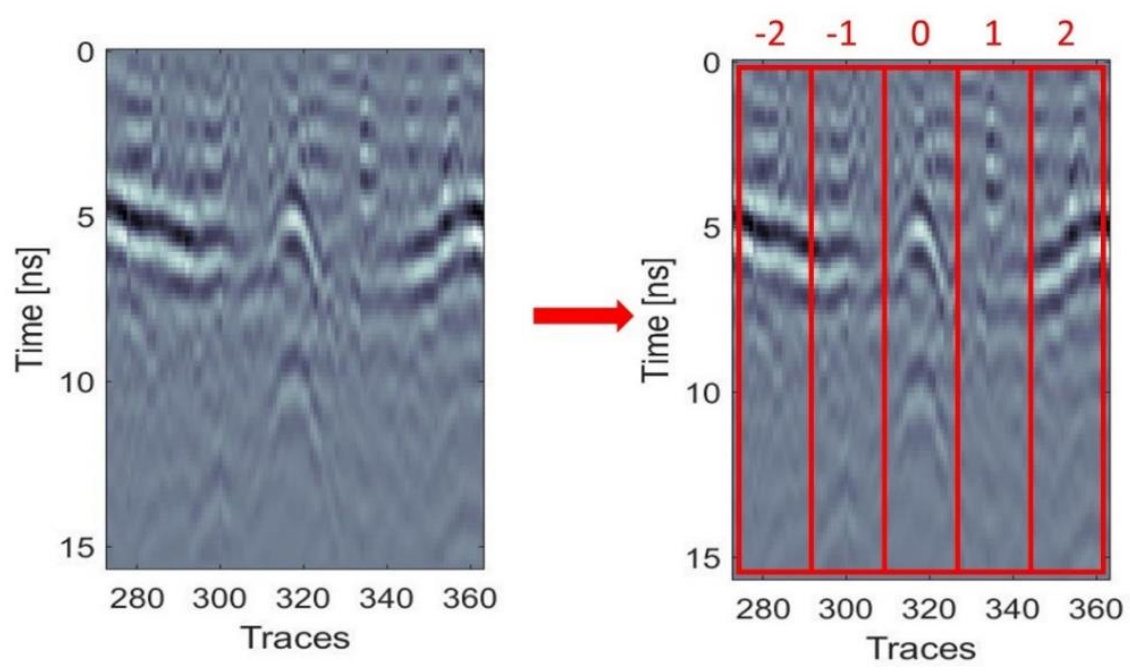

Figure 3. Subdivision of the selected area into application windows. 


\subsection{Application of the STFT Function}

Within each of the above-identified windows, the average value of the signal was calculated, which was subsequently used for the application of the STFT function. Figure 4 shows the time-frequency analysis carried out using the STFT approach.

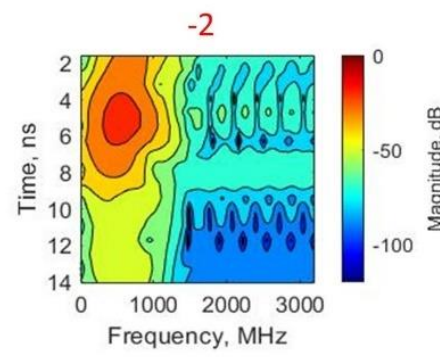

0

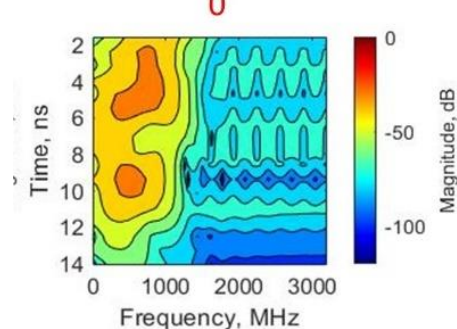

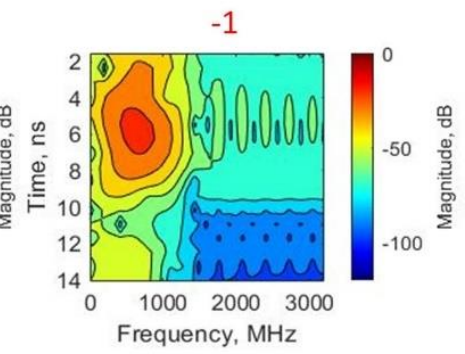

1

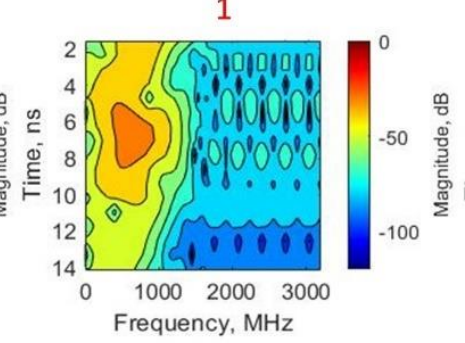

2

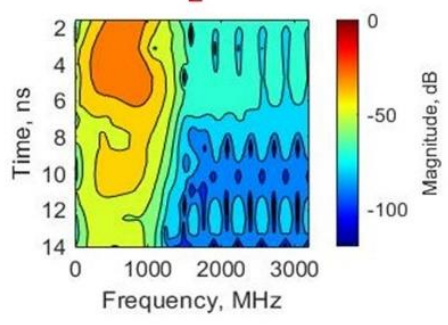

Figure 4. STFT spectra for the selected application windows

From the analysis of the STFT spectrum of the window 0, it can be seen how the energy is concentrated in two peaks, the first centred at an arrival time of about $5 \mathrm{~ns}$ and the second at an arrival time of about $10 \mathrm{~ns}$. This is then followed by an energy drop between 11 and $14 \mathrm{~ns}$. It is worth noting that the centroids of both areas are found in a frequency range between 500 and $700 \mathrm{MHz}$. Conversely, in the other windows, the energy is concentrated in a single peak, of greater intensity in windows -2 and -1 than in windows 1 and 2 . Consistently with the semi-horizontal trend of the layer, as highlighted in the B-scan, the centroids of the areas of greatest energy are located respectively at $5 \mathrm{~ns}$ for the -2 window, at $6 \mathrm{~ns}$ for the -1 window, at $7 \mathrm{~ns}$ for window 1 and $4 \mathrm{~ns}$ for window 2 . For all cases, the areas mentioned above are centred in a frequency range between 600 and $700 \mathrm{MHz}$. Unlike window 0, in all other cases, the energy is attenuated less rapidly.

\subsection{Error Maps}

To analyse in more detail the differences in the behaviour of the STFT spectra between the two cases (i.e. root or horizontal layer), error maps have been created, given by the difference between the elements in the examined map and those of the reference map. Figure 5 shows the error maps created for the selected application windows, with reference to the window 0 STFT spectra. 

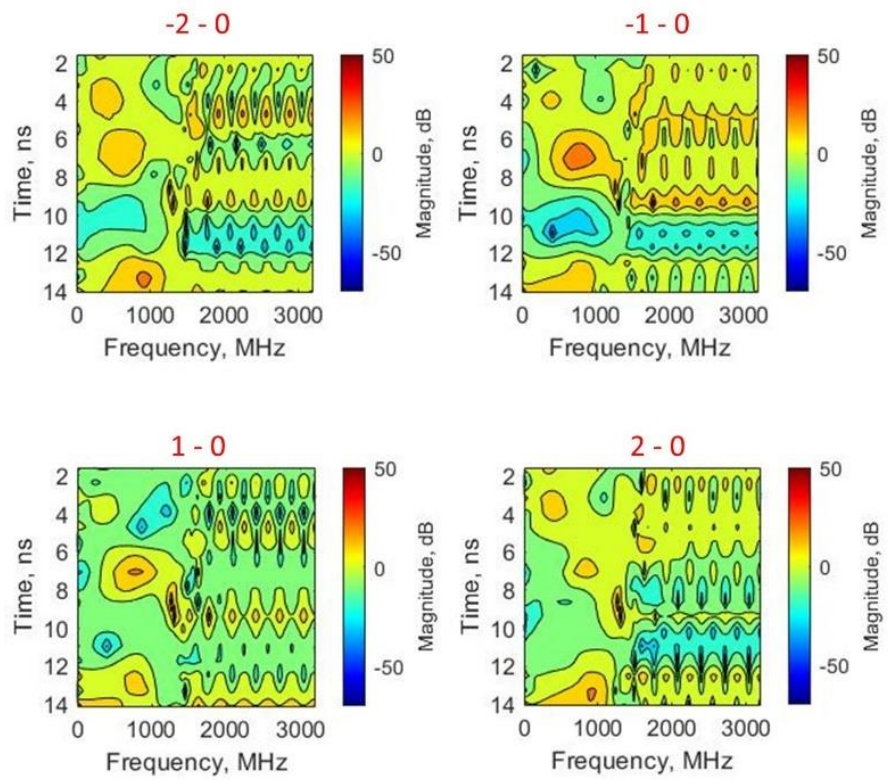

Figure 5. Error maps for the STFT spectra, with reference to the window 0 STFT spectra.

It can be noted how some characteristics occur in all the error maps. For example, the presence of an area of positive energy is recurrent, centred around the $1000 \mathrm{MHz}$ frequency, at an arrival time between 6 and $8 \mathrm{~ns}$. In the same way, it is possible to notice in all the error maps a negative peak, also centred around the $1000 \mathrm{MHz}$ frequency, at an arrival time between 4 and $5 \mathrm{~ns}$. In order to observe the trend of these characteristics, compared with the response of the time-frequency analysis in the presence of a root, the spectra of the energy in the error maps were evaluated. To this extent, a time window was selected, of width and depth equal to those of the reference hyperbola (i.e. between $4.5 \mathrm{~ns}$ and $5.5 \mathrm{~ns}$ ), as shown in Figure 6. The average spectra trend of the error maps within the selected time window is shown in Figure 7. It is worth noting that the error maps' average spectra all have the same trend, with an energy peak around $250 \mathrm{MHz}$ and a negative peak between 600 and $800 \mathrm{MHz}$.
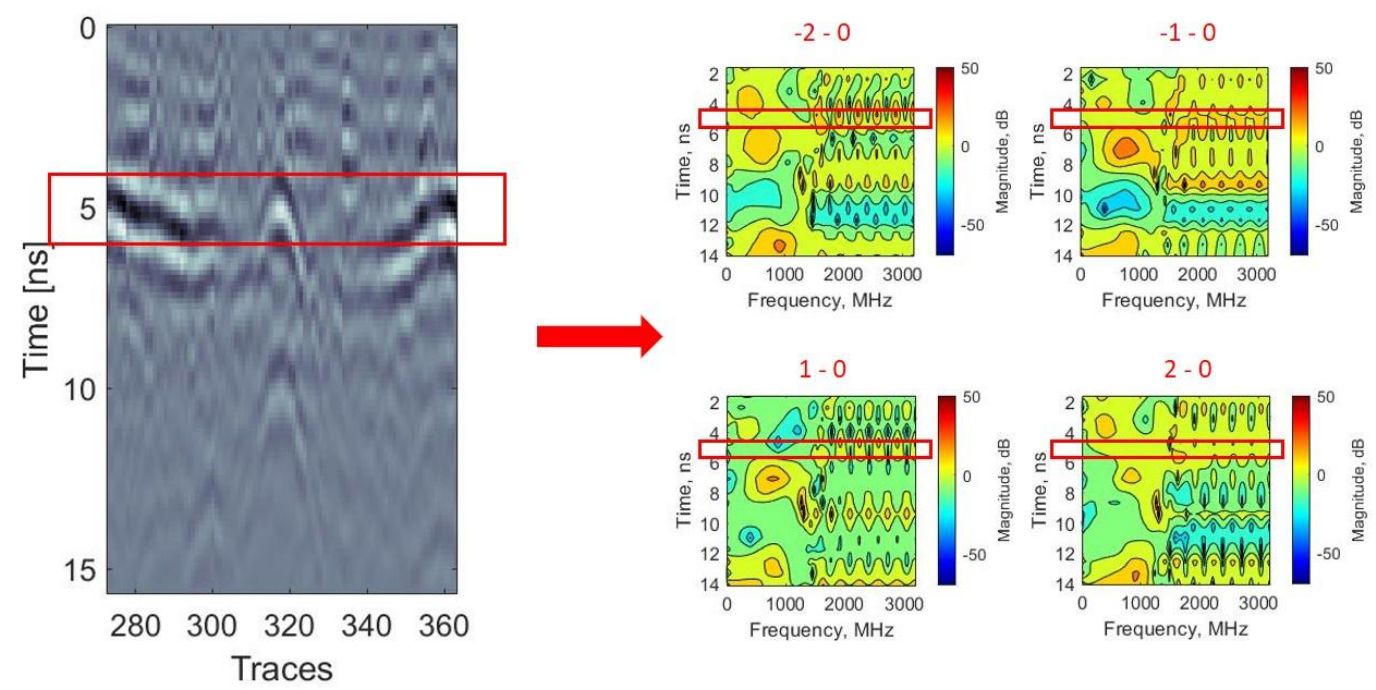

Figure 6. Selection of the time window for the analysis of the error maps' spectra trend 


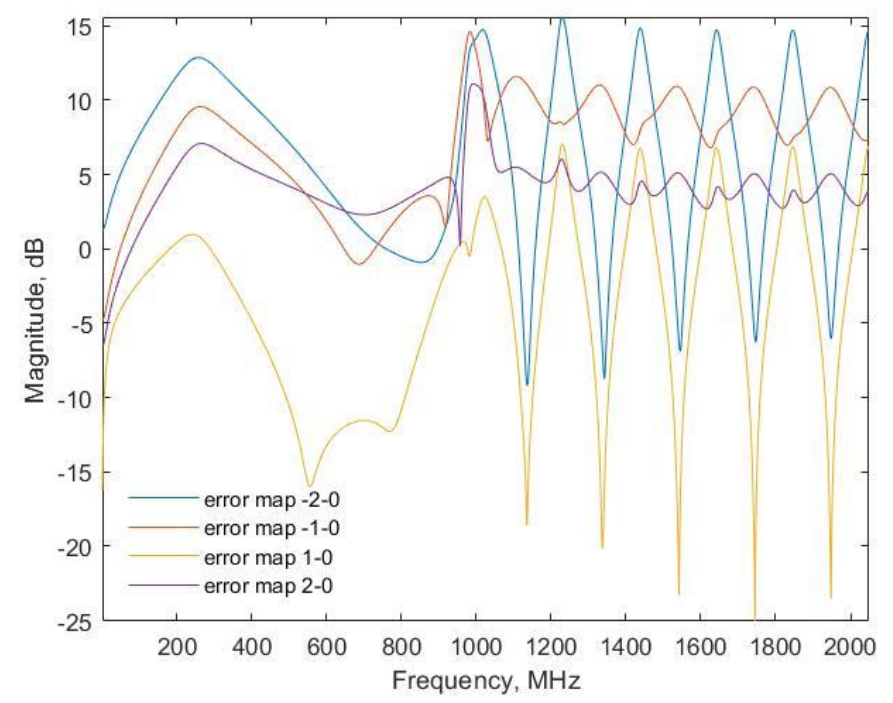

Figure 7. Error maps' average spectra trend between 4.5 and $5.5 \mathrm{~ns}$

\section{CONCLUSIONS AND FUTURE DEVELOPMENTS}

This study presents the preliminary outputs of novel research within the context of the applications of ground penetrating radar (GPR) in detecting and assessing tree roots systems. The primary aim of this research is to explore the feasibility of a new approach for tree root assessment, based on the time and frequency domain analysis of GPR data. To this extent, a data processing framework was established, where advanced signal processing techniques were applied to eliminate noiserelated information and the disturbance generated by the presence of other features.

Results' interpretation has shown the viability of the presented approach in recognising the influence of different features (i.e. roots, layers, etc.) on the combined time-frequency analysis of GPR data. Besides, the latter allowed the detection of recurring patterns in the data analysis, proving this method to be worthy for further development and implementation in tree root systems' assessment.

The preliminary results have shown the potential of this methodology which, if further investigated and automated, could be successfully used in urban areas, where some trees are difficult to access and to be thoroughly investigated. The presented methodology would allow for the development of an accurate, albeit expeditious, technology which would allow the protection of the urban natural heritage and the safeguarding of the built environment.

\section{REFERENCES}

[1] Pallardy, S. G., [Physiology of Woody Plants], Academic Press, Cambridge, Massachusetts (2010).

[2] Donovan, G. H., Butry, D. T., Michael, Y. L., Prestemon, J. P., Liebhold, A. M., Gatziolis, D. and Mao, M. Y., "The relationship between trees and human health: evidence from the spread of the emerald ash borer," American journal of preventive medicine 44(2), 139-145 (2013).

[3] van Dillen, Sonja M E, de Vries, S., Groenewegen, P. P. and Spreeuwenberg, P., "Greenspace in urban neighbourhoods and residents' health: adding quality to quantity," Journal of Epidemiology and Community Health 66(6) (2012).

[4] Mullaney, J., Lucke, T. and Trueman, S. J., "A review of benefits and challenges in growing street trees in paved urban environments," Landscape and Urban Planning 134, 157-166 (2015).

[5] Tallis, M., Taylor, G., Sinnett, D. and Freer-Smith, P., "Estimating the removal of atmospheric particulate pollution by the urban tree canopy of London, under current and future environments," Landscape and Urban Planning 103(2), 129-138 (2011). 
[6] Tyrväinen, L., Pauleit, S., Seeland, K. and de Vries, S. , "Benefits and Uses of Urban Forests and Trees,", 81-114 (2005).

[7] Coutts, M. P., "Root architecture and tree stability," Plant and Soil 71(1/3), 171-188 (1983).

[8] Wilson, B. F., [Structure and Growth of Woody Roots of Acer Rubrum L], Harvard University, Harvard Forest St. Petersham (1964).

[9] Stokes, A., Fitter, A. H. and Courts, M. P., "Responses of young trees to wind and shading: effects on root architecture," J.Exp.Bot. 46(9), 1139-1146 (1995).

[10] Innes, J. L., [Forest Health: Its Assessment and Status.], CAB international (1993).

[11] Reubens, B., Poesen, J., Danjon, F., Geudens, G. and Muys, B., "The role of fine and coarse roots in shallow slope stability and soil erosion control with a focus on root system architecture: a review," Trees 21(4), 385-402 (2007).

[12] Guo, L., Chen, J., Cui, X., Fan, B. and Lin, H., "Application of ground penetrating radar for coarse root detection and quantification: a review," Plant Soil 362(1-2), 1-23 (2013).

[13] Čermák, J., Nadezhdina, N., Meiresonne, L. and Ceulemans, R., "Scots pine root distribution derived from radial sap flow patterns in stems of large leaning trees," Plant Soil 305(1-2), 61-75 (2008).

[14] Gregory, P. J., Hutchison, D. J., Read, D. B., Jenneson, P. M., Gilboy, W. B. and Morton, E. J., "Non-invasive imaging of roots with high resolution X-ray micro-tomography," 101, 351-359 (2003).

[15] Moran, C. J., Pierret, A. and Stevenson, A. W., "X-ray absorption and phase contrast imaging to study the interplay between plant roots and soil structure," Plant Soil 223(1-2), 101-117 (2000).

[16] Paglis, C. M., "Application of electrical resistivity tomography for detecting root biomass in coffee trees," International Journal of Geophysics 2013, 6 (2013).

[17] Amato, M., Basso, B., Celano, G., Bitella, G., Morelli, G. and Rossi, R., "In situ detection of tree root distribution and biomass by multi-electrode resistivity imaging," Tree Physiol. 28(10), 1441-1448 (2008).

[18] Tosti, F., Bianchini Ciampoli, L., D'Amico, F., Alani, A. M. and Benedetto, A., "An experimental-based model for the assessment of the mechanical properties of road pavements using ground-penetrating radar," Construction \& building materials 165, 966-974 (2018).

[19] Giannakis, I., Giannopoulos, A. and Warren, C., "A realistic FDTD numerical modeling framework of ground penetrating radar for landmine detection," IEEE journal of selected topics in applied earth observations and remote sensing 9(1), 37-51 (2015).

[20] Daniels, D. J., [Ground Penetrating Radar], The Institution of Electrical Engineers, London (2004).

[21] Hruska, J., Čermák, J. and Šustek, S., "Mapping tree root systems with ground-penetrating radar," Tree Physiol.

19(2), 125-130 (1999).

[22] Barton, C. V. M. and Montagu, K. D., "Detection of tree roots and determination of root diameters by ground penetrating radar under optimal conditions," Tree physiology 24(12), 1323-1331 (2004).

[23] Butnor, J. R., Doolittle, J. A., Johnsen, K. H., Samuelson, L., Stokes, T. and Kress, L., "Utility of groundpenetrating radar as a root biomass survey tool in forest systems," Soil Sci.Soc.Am.J. 67(5), 1607-1615 (2003).

[24] Alani, A. M., Ciampoli, L. B., Lantini, L., Tosti, F. and Benedetto, A., "Mapping the root system of matured trees using ground penetrating radar," 2018 17th International Conference on Ground Penetrating Radar (GPR), 1-6 (2018).

[25] Lantini, L., Holleworth, R., Egyir, D., Giannakis, I., Tosti, F. and Alani, A. M., "Use of Ground Penetrating Radar for Assessing Interconnections between Root Systems of Different Matured Tree Species," 2018 Metrology for Archaeology and Cultural Heritage (MetroArchaeo), 22-26 (2018).

[26] Lantini, L., Tosti, F., Giannakis, I., Zou, L., Benedetto, A. and Alani, A. M., "An Enhanced Data Processing Framework for Mapping Tree Root Systems Using Ground Penetrating Radar," Remote Sensing 12(20), 3417 (2020).

[27] Lantini, L., Alani, A., Giannakis, I., Benedetto, A. and Tosti, F., "Application of ground penetrating radar for mapping tree root system architecture and mass density of street trees," Advances in Transportation Studies(3), 51-62 (2020).

[28] Benedetto, A., Tosti, F., Bianchini Ciampoli, L. and D’Amico, F., "An overview of ground-penetrating radar signal processing techniques for road inspections," Signal Processing 132, 201-209 (2017).

[29] Kim, J., Cho, S. and Yi, M., "Removal of ringing noise in GPR data by signal processing," Geosci J 11(1), 75-81 (2007).

[30] Bianchini Ciampoli, L., Calvi, A. and D'Amico, F., "Railway Ballast Monitoring by Gpr: A Test Site Investigation," Remote Sensing 11(20), 2381 (2019). 